\title{
Incidence and predictors of 30-day hospital readmissions for liver cirrhosis: insights from the United States National Readmissions Database
}

\author{
Sushil Kumar Garg ${ }^{1 \#}$, Hemant Goyal ${ }^{2 \#}$, Itegbemie Obaitan ${ }^{3}$, Pir Ahmad Shah ${ }^{4}$, Shashank Sarvepalli ${ }^{5}$, \\ Loretta Lynn Jophlin ${ }^{6}$, Dupinder Singh ${ }^{3}$, Sumeet Asrani ${ }^{7}$, Patrick S. Kamath ${ }^{1}$, Michael D. Leise ${ }^{1}$ \\ ${ }^{1}$ Division of Gastroenterology and Hepatology, Mayo Clinic, Rochester, MN, USA; ${ }^{2}$ Mercer University School of Medicine, Macon, GA, USA; \\ ${ }^{3}$ Department of Gastroenterology and Hepatology, University of Minnesota, Minneapolis, MN, USA; ${ }^{4}$ Beth Israel Deaconess Medical Center, \\ Boston, MA, USA; ${ }^{5}$ Department of Hospital Medicine, Cleveland Clinic, Cleveland, OH, USA; ${ }^{6}$ Division of Gastroenterology-Hepatology, \\ University of Nebraska Medical Center, Omaha, NE, USA; ${ }^{7}$ Baylor University Medical Center, Baylor Scott and White, Dallas, TX, USA \\ Contributions: (I) Conception and design: All authors; (II) Administrative support: All authors; (III) Provision of study materials or patients: All \\ authors; (IV) Collection and assembly of data: All authors; (V) Data analysis and interpretation: SK Garg; (VI) Manuscript writing: All authors; (VII) \\ Final approval of manuscript: All authors. \\ \#These authors contributed equally to this work. \\ Correspondence to: Michael D. Leise, MD. Division of Gastroenterology and Hepatology, Mayo Clinic, 200 First St SW, Rochester, MN 55905, USA. \\ Email: leise.michael@mayo.edu.
}

Background: Cirrhosis is associated with substantial inpatient morbidity and mortality. This study aimed to determine the trends in 30-day hospital readmission rates among patients with cirrhosis and identify factors associated with these readmissions.

Methods: We conducted a retrospective analysis of data retrieved from the Nationwide Readmissions Database to determine trends in 30-day readmission for patients discharged with a diagnosis of cirrhosis in 2010 through 2014. Multivariate logistic regression analysis was used to identify predictors of readmission.

Results: Among 303,346 patients identified from the database, the 30-day readmission rate for patients with a discharge diagnosis of cirrhosis was 31.4\% ( $n=95,298)$. The trends in the readmission rates remained steady during the study period. On multivariate analysis, female sex, age 45 years or older, esophagogastroduodenoscopy (EGD) during admission, and disposition to a short-term care facility or skilled nursing facility protected against readmissions. In contrast, coverage by Medicaid insurance, admission during a weekend, nonalcoholic cause of cirrhosis, and history of hepatic encephalopathy and ascites were associated with readmission.

Conclusions: We found an exceptionally high 30-day readmission rate in patients with cirrhosis, although it remained stable during the study period. This study identified some modifiable factors such as disposition to a short-term care facility or skilled nursing facility and patients' attendance of alcohol rehabilitation facilities that could decrease the likelihood of readmission and could inform local and national healthcare policymakers.

Keywords: Cirrhosis; national readmission database; 30-day readmission; healthcare cost

Submitted Feb 20, 2020. Accepted for publication May 23, 2021.

doi: 10.21037/atm-20-1762

View this article at: https://dx.doi.org/10.21037/atm-20-1762 


\section{Introduction}

Liver disease is responsible for approximately two million deaths worldwide every year, of which about half are due to the complications related to cirrhosis, and the other half are due to viral hepatitis and liver cancer (1). In the United States (US), the prevalence of cirrhosis has been reported to be $0.27 \%$, with a mortality rate of $26.4 \%$ (2). Despite the substantial advances in the medical and pharmacologic therapy, the inpatient burden associated with cirrhosis has continued to increase annually, with the number of hospitalizations reaching 658,900 in 2011 (3). From 2001 to 2011, the annual cost of cirrhosis-related hospitalizations doubled from $\$ 4.8$ billion to $\$ 9.8$ billion, and the mean cost of hospitalization increased from $\$ 13,079$ to $\$ 15,193$ (3). A recent systematic review showed that the hospitalization rate for cirrhosis or complications of portal hypertension increased by 31\% between 1993 and 2012 (4). A Texas state hospitalization database study revealed that rate of chronic liver disease (CLD) related hospitalizations increased by $92 \%$ from 2004 to 2013 (from 1,295 to 2,490/100,000 hospitalizations), as compared to $48.8 \%$ for chronic obstructive pulmonary diseases (COPD) and only $6.7 \%$ for congestive heart failure (CHF) (5). In fact, hospitalization costs associated with cirrhosis have been reported to be even higher than those for CHF or COPD (6).

Readmission after a hospital stay is a significant contributor to healthcare costs and may be preventable. Early readmission, within the first few days after the initial discharge, has been reported to be unusually high for some conditions and places a substantial economic burden on the healthcare system (7-9). The rate of readmission for cirrhosis is high and is associated with a significant economic impact. A database study from Texas showed that patients with CLD have a higher 30-day re-hospitalization rate $(25 \%)$ than patients with $\mathrm{CHF}(21.9 \%)$ and COPD (20.6\%) (5). Similarly, a recent study from the State Inpatient Database for California reported that the 30-day readmission rate for cirrhosis was as high as $18.8 \%$ between 2009 and 2011 (10). In a single-center analysis of 222 patients with decompensated cirrhosis from January 2011 to December 2013, the 30- and 90-day readmission rates were $20.7 \%$ and $30.1 \%$, respectively (11). A retrospective analysis of 122 Veterans Administration hospitals between 2010 and 2013 reported that $13.8 \%$ of patients were readmitted, and $4.6 \%$ died between 8 and 30 days after discharge. Another small, single-center study reported that $42.4 \%$ of patients who underwent paracentesis required early readmission, which is an alarmingly high proportion (12). Besides its associated economic burden, readmission in patients with cirrhosis is also an independent risk factor for death $(2,13)$.

Although hospitalizations account for a substantial portion of healthcare utilization among patients with cirrhosis, relatively little is known about the epidemiology and predictors of 30-day hospital readmission for cirrhosis in the US. Most of the previous studies on readmission of patients with cirrhosis focused on select patient populations: single centers (11), states (10), regions (14), and ethnic group analyses (15). In the current study, we examined an all-payer (including uninsured) nationally representative readmission database to determine the trends in and incidence of 30-day readmissions for patients with cirrhosis. Additionally, we identified factors associated with these readmissions. We present the following article in accordance with the STROBE reporting checklist (available at https://dx.doi.org/10.21037/atm-20-1762).

\section{Methods}

\section{Study design}

We conducted a retrospective analysis of data obtained for patients with a primary diagnosis of liver cirrhosis based on the International Classification of Diseases, Ninth Revision, Clinical Modification (ICD-9-CM) codes (571.2: alcoholic cirrhosis of liver; 571.5: cirrhosis of liver without mention of alcohol; and 571.6: biliary cirrhosis), who were discharged from hospitals between January 2010 and December 2014.

\section{Data source}

The data was collected from the Nationwide Readmissions Database (NRD), which is the largest nationally representative inpatient database and is maintained by the Agency for Healthcare Research and Quality (AHRQ) under the Healthcare Cost and Utilization Project (HCUP). The NRD is a unique, all-payer (including uninsured) inpatient database that represents approximately $49.3 \%$ of all the hospitalizations occurring in 27 geographically diverse states in the US. It provides data from approximately 36 million discharges across the US. The NRD covers data on more than 100 clinical and nonclinical variables and makes these data available in an anonymized manner. These variables include data on demographic characteristics, length of stay (LOS), the interval between consecutive 


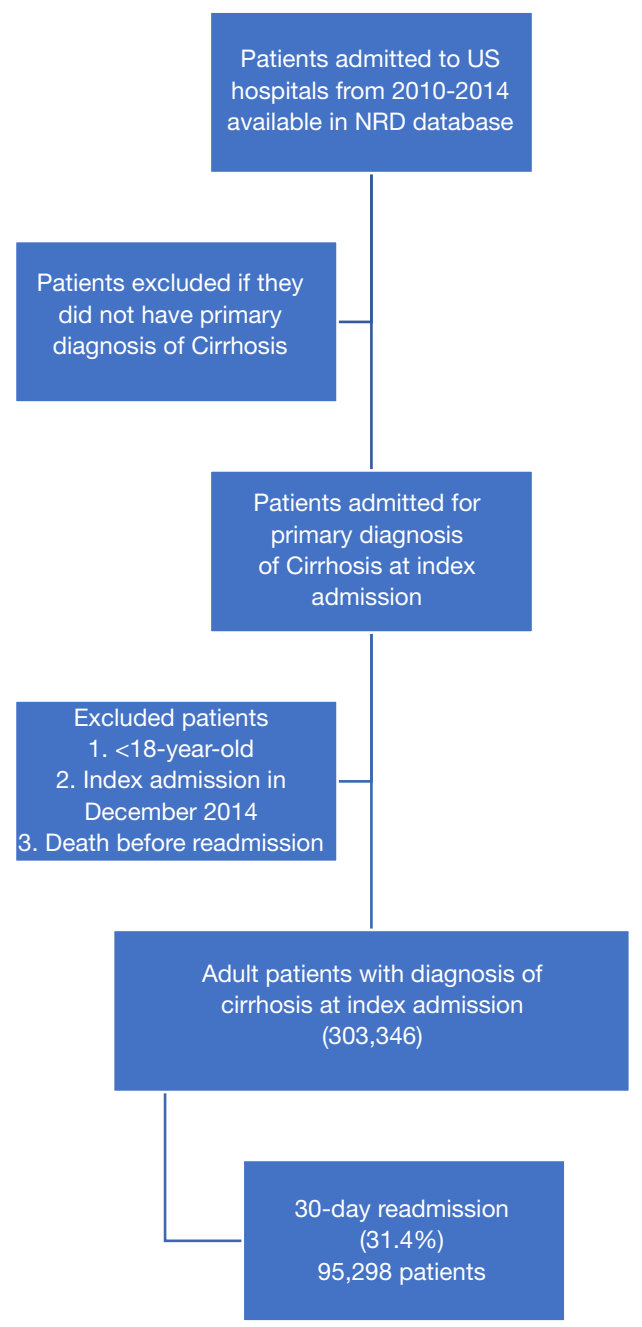

Figure 1 Prima diagram for patient selection.

admissions, expected pay source, hospital costs and total charges, and diagnostic and procedure codes based on the ICD-9-CM coding.

The data were collected for the following parameters: hospital bed size, teaching status of the hospital, patients' demographic and clinical features, payer status, household income, disposition, Charlson Comorbidity Index (CCI), admission day (weekend or weekday), duration of hospitalization, status of alcohol rehabilitation, presence of complications [e.g., hepatic encephalopathy (HE), ascites, hepatorenal syndrome (HRS)]. Data on procedures performed, including esophagogastroduodenoscopy (EGD), thoracocentesis, paracentesis, blood transfusion, transjugular intrahepatic portosystemic shunt (TIPS) placement, and bariatric surgery were also collected.

\section{Study population}

From the NRD we retrieved data on all adult patients (age $\geq 18$ years) who met the inclusion criteria of hospital discharge with primary diagnosis of cirrhosis (ICD-9-CM codes 571.2, 571.5, and 571.6) during the index hospital admission from January 2010 through November 2014 and who were readmitted within 30 days after discharge from the index admission. The first hospitalization during the study period was considered the patient's index admission. All index admissions during December 2014 were excluded from the study because subsequent 30-day follow-up data were not analyzed in these cases. Furthermore, if a patient had more than one readmission within 30 days of the initial discharge, only the first episode of readmission was included in the analysis (Figure 1). The study was conducted in accordance with the Declaration of Helsinki (as revised in 2013).

\section{Definition of variables, comorbid conditions, and other covariates}

We obtained demographic characteristics, including patients' age, sex, the median income per home postal code, and insurance data. ICD-9-CM diagnostic codes were used to collect information regarding comorbid conditions, discharge diagnoses, procedures performed, and discharge disposition (Appendix 1). The CCI was used for comorbidity assessment. Hospital information, such as hospital size, teaching status, and location was also collected.

\section{Statistical analysis}

Categorical variables were reported as the frequency of occurrence. The $\chi^{2}$ test was used to assess the significance of these differences. A logistic regression model was used to determine predictors of readmission within 30 days. Data were reported as odds ratio (OR) and 95\% CI. The analysis was done using discharge-level weight (DISCWT) to produce national estimates. All statistical analyses were performed using SAS software version 9.4 (SAS Institute Inc.). $\mathrm{P}$ values $<0.05$ were considered statistically significant.

\section{Results}

Overall, there were 303,346 patients with a primary diagnosis of cirrhosis on index hospitalization during the study period. Of these, 95,298 patients (31.4\%) were 


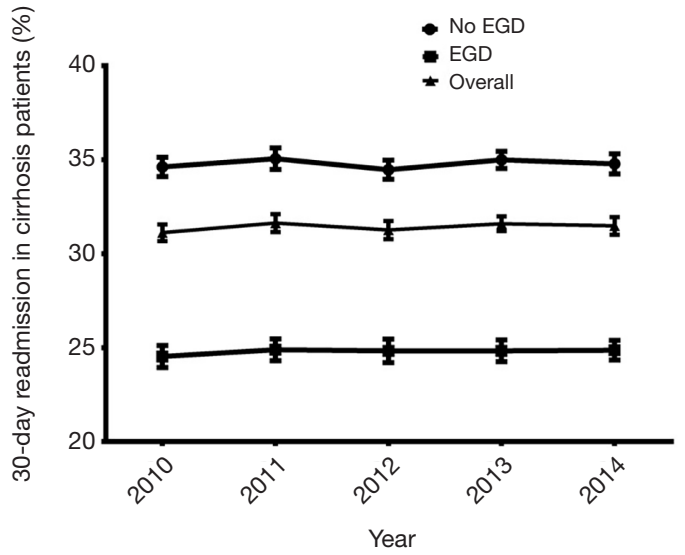

Figure 2 30-day readmission in cirrhosis patients.

readmitted within 30 days after discharge for cirrhosisrelated complications coded as ICD-9 (456.0, 456.1, 572.2, $572.3,572.4$ and 789.59). The rate of readmission over the 5 -year study period remained stable (Figure 2).

\section{Univariate analysis}

The baseline demographic characteristics of patients with and without readmission were analyzed (Table 1). On univariate analysis, patient age $(\mathrm{P}<0.001)$, weekend/weekday admission $(\mathrm{P}=0.01)$, median household income $(\mathrm{P}=0.02)$, payer type $(\mathrm{P}<0.001)$, and disposition status $(\mathrm{P}<0.001)$, as well as hospital control $(\mathrm{P}=0.008)$, and teaching status $(\mathrm{P}<0.001)$ were significantly different between patients with and without readmission (Table 2). Patients with readmission also had higher incidences of associated secondary diagnoses of acute kidney injury, HRS, ascites, HE, esophageal varices without bleeding, and nonalcoholic cirrhosis (all $\mathrm{P}<0.001$ ), as well as higher requirement for procedures such as paracentesis $(\mathrm{P}<0.001)$, thoracentesis $(\mathrm{P}<0.001)$, TIPS $(\mathrm{P}=0.002)$ during the index admission. In addition, patients who were readmitted within 30 days had higher rates of comorbid conditions such as CHF $(\mathrm{P}<0.001)$, COPD $(\mathrm{P}<0.001)$, diabetes mellitus $(\mathrm{P}<0.001)$, renal disease $(\mathrm{P}<0.001)$, peripheral vascular disease $(\mathrm{P}<0.001)$, and cancer $(\mathrm{P}<0.001)$ (Table 3).

\section{Multivariate analysis}

When patients' demographic characteristics were assessed with multivariate analysis for factors associated with readmission (Table 4), women were less likely than men to be readmitted for cirrhosis (OR, 0.95; 95\% CI, 0.93-0.98; $\mathrm{P}=0.002$ ).
Interestingly, compared with patients aged 18-44 years, patients in other age groups had lower odds of readmission, with the risk being the lowest among patients older than 84 years (OR, 0.51; 95\% CI, 0.46-0.58; $\mathrm{P}<0.001)$. Regarding payer type, patients with private insurance (OR, 0.76; $95 \%$ CI, 0.73-0.80; $\mathrm{P}<0.001)$ and those who were uninsured or had other types of insurance (OR, 0.64; 95\% CI, 0.61-0.67; $\mathrm{P}<0.001$ ) had decreased odds of readmission compared with patients with Medicare insurance. Patients with Medicaid insurance, however, had a higher odd of readmission (OR, 1.09; 95\% CI, 1.04-1.13; $\mathrm{P}<0.001)$ than the Medicare population. There was no influence of median household income or size of the hospital (number of beds) on readmission rate.

Other factors associated with a high risk of 30-day readmission were being admitted over a weekend (OR, 1.08; 95\% CI, 1.04-1.11; $\mathrm{P}<0.001)$, CCI score greater than 1 (OR, $1.07 ; 95 \% \mathrm{CI}, 1.02-1.11 ; \mathrm{P}=0.002)$, and presence of $\mathrm{HE}(\mathrm{OR}$, 1.22 ; 95\% CI, 1.18-1.27; $\mathrm{P}<0.001$ ), HRS (OR, 1.14; $95 \% \mathrm{CI}$, 1.06-1.23; $\mathrm{P}<0.001$ ), ascites (OR, 1.44; 95\% CI, 1.39-1.49; $\mathrm{P}<0.001)$, and esophageal varices without bleeding (OR, 1.07; 95\% CI, 1.02-1.11; $\mathrm{P}=0.003)$. The results indicated that discharge to a short-term hospital (OR, 0.71; 95\% CI, $0.63-$ $0.81 ; \mathrm{P}<0.001)$ or skilled nursing facility (OR, $0.86 ; 95 \%$ CI, 0.82-0.91; $\mathrm{P}<0.001$ ) was associated with a lower odds of readmission as compared with being discharged home. However, leaving against medical advice increased the odds of readmission (OR, 1.59; 95\% CI, 1.47-1.72; $\mathrm{P}<0.001$ ).

Furthermore, the 30-day readmission rate was lower among patients who had EGD than among those who had not undergone EGD during the index admission (OR, 0.79; 95\% CI, 0.76-0.83; $\mathrm{P}<0.001$ ) (Figure 1). With respect to the type of cirrhosis, patients with biliary cirrhosis (OR, 1.26; 95\% CI, 1.11-1.44; $\mathrm{P}<0.001$ ) or nonalcoholic cirrhosis (OR, 1.15; 95\% CI, 1.12-1.19; $\mathrm{P}<0.001$ ) had higher rates of early readmissions than those with alcoholic cirrhosis (Figure 1). Patients who participated in alcohol rehabilitation programs (OR, 0.86; 95\% CI, 0.78-0.96; $\mathrm{P}=0.006$ ) had lower readmission risk than those who did not. The odds of readmission were not increased because of obesity (OR, 0.96; 95\% CI, 0.89-1.02; $\mathrm{P}=0.20$ ), but patients with previous bariatric surgery had increased odds of readmission (OR, 1.32; 95\% CI, 1.14-1.53; $\mathrm{P}<0.001$ ).

\section{Causes of readmission}

The most frequent primary causes of readmission in patients with cirrhosis during the index hospitalization are shown in Table 5. The two most common reasons for 
Table 1 Baseline demographic and clinical characteristics of patients with cirrhosis, stratified by readmission status ${ }^{a}$

\begin{tabular}{|c|c|c|c|c|}
\hline Characteristic & All patients $(\mathrm{N}=303,346)$ & \multicolumn{2}{|c|}{ Readmission } & $P$ value \\
\hline Men & $194,978(64.3)$ & $133,857(64.3)$ & $61,122(64.1)$ & 0.57 \\
\hline Age, y & & & & $<0.001$ \\
\hline $18-44$ & $37,640(12.4)$ & $25,300(12.2)$ & $12,341(12.9)$ & \\
\hline $65-84$ & $65,376(21.6)$ & $45,339(21.8)$ & $20,037(21.0)$ & \\
\hline$>84$ & $5,370(1.8)$ & $4,043(1.9)$ & $1,327(1.4)$ & \\
\hline Weekend admission & $66,006(21.8)$ & $44,819(21.5)$ & $21,187(22.2)$ & 0.01 \\
\hline Disposition & & & & $<0.001$ \\
\hline Skilled nursing facility & $34,031(11.2)$ & $23,718(11.4)$ & $10,313(10.8)$ & \\
\hline Home health care & 41,109 (13.6) & $27,229(13.1)$ & $13,881(14.6)$ & \\
\hline Against medical advice & $7,962(2.6)$ & $4,465(2.1)$ & $3,497(3.7)$ & \\
\hline \multicolumn{5}{|l|}{ Secondary diagnoses } \\
\hline ICU stay & $5,706(1.9)$ & $4,239(2.0)$ & $1,467(1.5)$ & $<0.001$ \\
\hline Acute kidney injury & $51,892(17.1)$ & $32,653(15.7)$ & $19,239(20.2)$ & $<0.001$ \\
\hline Acute respiratory failure & $18,651(6.1)$ & $13,149(6.3)$ & $5,502(5.8)$ & $<0.001$ \\
\hline Pneumonia & $11,569(3.8)$ & $7,911(3.8)$ & $3,658(3.8)$ & 0.78 \\
\hline Esophageal varices (with bleeding) & $71,012(23.4)$ & $55,051(26.5)$ & $15,961(16.7)$ & $<0.001$ \\
\hline Esophageal varices (without bleeding) & $51,118(16.9)$ & $33,733(16.2)$ & $17,386(18.2)$ & $<0.001$ \\
\hline \multicolumn{5}{|l|}{ Procedures } \\
\hline Thoracentesis & $9,620(3.2)$ & $5,881(2.8)$ & $3,738(3.9)$ & $<0.001$ \\
\hline TIPS & $10,694(3.5)$ & $6,983(3.4)$ & $3,711(3.9)$ & 0.002 \\
\hline Paracentesis & $151,712(50.0)$ & $9,6518(46.4)$ & $55,194(57.9)$ & $<0.001$ \\
\hline Esophagogastroduodenoscopy & $101,855(33.6)$ & $76,619(36.8)$ & $25,235(26.5)$ & $<0.001$ \\
\hline Weight & & & & 0.08 \\
\hline No obesity & $276,878(91.3)$ & $189,957(91.3)$ & $86,921(91.2)$ & \\
\hline Obesity & $15,405(5.1)$ & $10,683(5.1)$ & $4,722(5.0)$ & \\
\hline Morbid obesity & $11,063(3.6)$ & $7,409(3.6)$ & $3,654(3.8)$ & \\
\hline
\end{tabular}

Table 1 (continued) 
Table 1 (continued)

\begin{tabular}{lccc}
\hline Characteristic & All patients $(\mathrm{N}=303,346)$ & Readmission \\
\cline { 3 - 4 } & & No $(\mathrm{n}=208,049)$ & Yes $(\mathrm{n}=95,298)$ \\
\hline Cirrhosis & $189,598(62.5)$ & $131,850(63.4)$ & $57,748(60.6)$ \\
Alcoholic cirrhosis & $108,842(35.9)$ & $72,949(35.1)$ & $35,893(37.7)$ \\
Cirrhosis without mention of alcohol & $4,906(1.6)$ & $3,249(1.6)$ & $1,657(1.7)$ \\
Biliary cirrhosis & & & \\
\hline
\end{tabular}

${ }^{a}$ Values are No. of patients (\%). ICU, intensive care unit; TIPS, transjugular intrahepatic portosystemic shunt.

Table 2 Baseline hospital-related and socioeconomic characteristics of patients with cirrhosis, stratified by readmission status ${ }^{\mathrm{a}}$

\begin{tabular}{|c|c|c|c|c|}
\hline Characteristic & All patients $(\mathrm{N}=303,346)$ & \multicolumn{2}{|c|}{ Readmission } & $P$ value \\
\hline Hospital size & & & & 0.26 \\
\hline Small & $30,005(9.9)$ & $20,827(10.0)$ & $9,178(9.6)$ & \\
\hline Medium & $70,752(23.3)$ & $48,654(23.4)$ & 22,099 (23.2) & \\
\hline Hospital control & & & & 0.008 \\
\hline Government & $47,946(15.8)$ & $32,831(15.8)$ & $15,116(15.9)$ & \\
\hline Private, not-for-profit & 206,398 (68.0) & $142,249(68.4)$ & $64,149(67.3)$ & \\
\hline Private, for-profit & $49,002(16.2)$ & $32,969(15.8)$ & $16,033(16.8)$ & \\
\hline Metropolitan teaching & $170,385(56.2)$ & 115,635 (55.6) & $54,749(57.5)$ & \\
\hline Nonmetropolitan & $23,399(7.7)$ & $16,706(8.0)$ & $6,692(7.0)$ & \\
\hline Median household income percentile based on ZIP code & & & & 0.02 \\
\hline 0th-25th & $101,439(34.1)$ & $68,888(33.8)$ & $32,551(34.8)$ & \\
\hline 26th-50th & $79,083(26.6)$ & $54,743(26.9)$ & $24,341(26.0)$ & \\
\hline 51st-75th & $67,834(22.8)$ & $46,585(22.9)$ & $21,249(22.7)$ & \\
\hline 76th-100th & $48,955(16.5)$ & $33,655(16.5)$ & $15,299(16.4)$ & \\
\hline Payer & & & & $<0.001$ \\
\hline
\end{tabular}


Table 3 Common comorbid conditions for patients with cirrhosis, stratified by readmission status ${ }^{\mathrm{a}}$

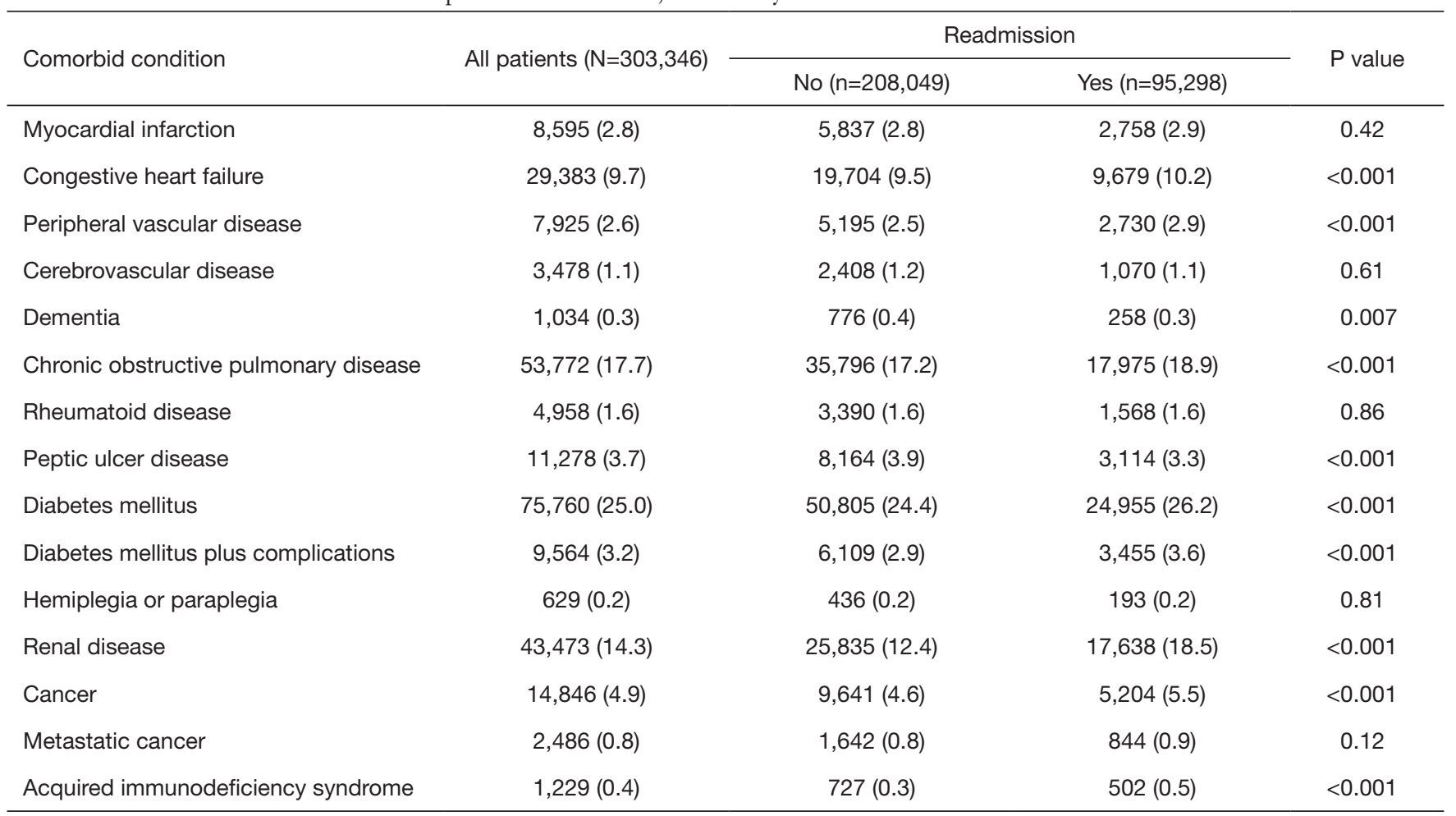

${ }^{a}$ Values are No. of patients (\%).

Table 4 Multivariate analysis of factors associated with early readmission for cirrhosis

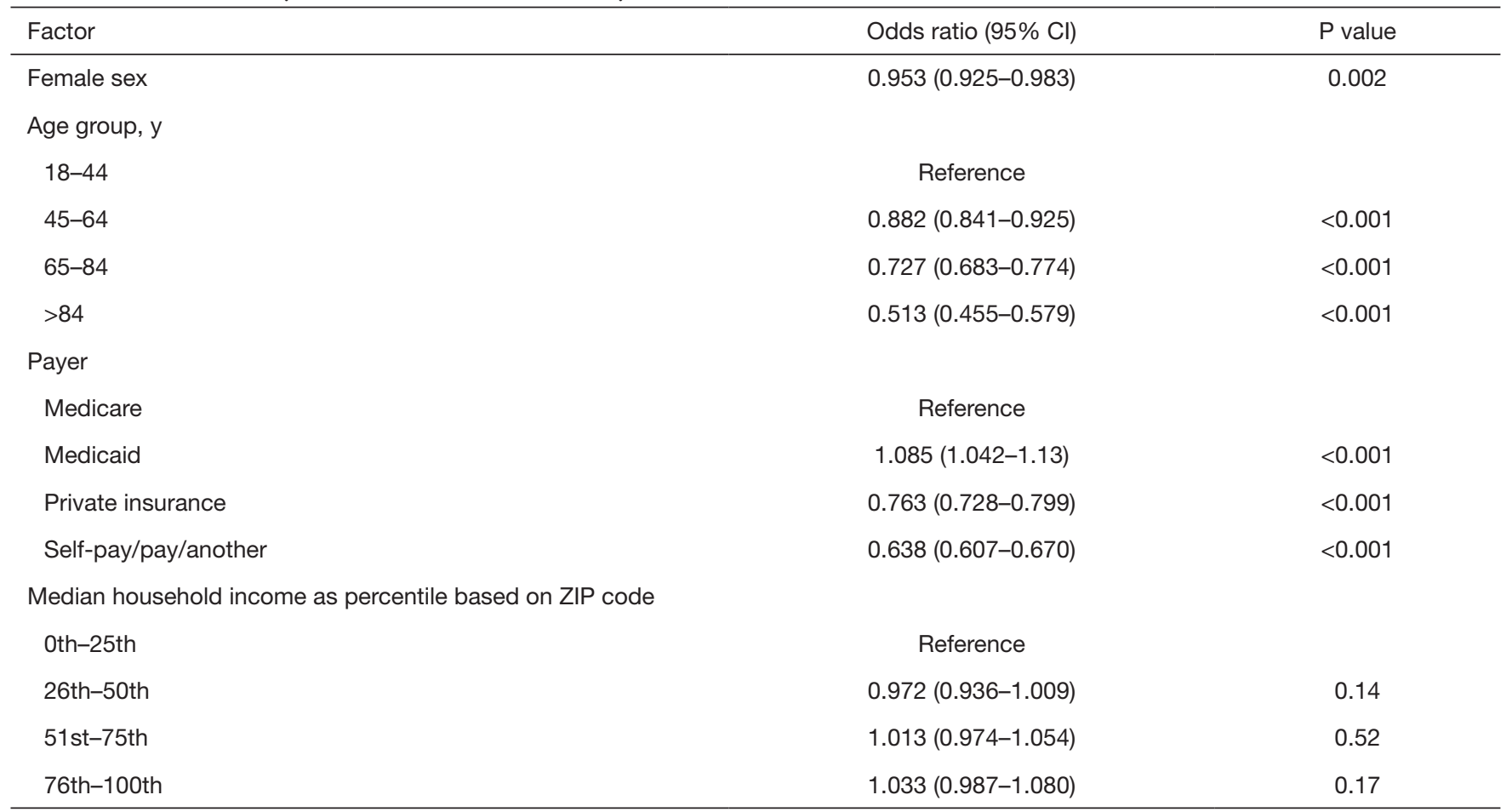

Table 4 (continued) 
Table 4 (continued)

\begin{tabular}{|c|c|c|}
\hline Factor & Odds ratio $(95 \% \mathrm{Cl})$ & $P$ value \\
\hline \multicolumn{3}{|l|}{ Hospital size } \\
\hline Small & Reference & \\
\hline Medium & $1.028(0.966-1.095)$ & 0.38 \\
\hline \multicolumn{3}{|l|}{ Disposition } \\
\hline Routine & Reference & \\
\hline Short-term hospital & $0.714(0.627-0.813)$ & $<0.001$ \\
\hline Skilled nursing facility & $0.861(0.816-0.910)$ & $<0.001$ \\
\hline Not obese & Reference & \\
\hline Obese & $0.957(0.894-1.024)$ & 0.20 \\
\hline Morbidly obese & $0.994(0.921-1.071)$ & 0.87 \\
\hline Charlson Comorbidity Index (>1 vs. 1) & $1.068(1.024-1.114)$ & 0.002 \\
\hline Hepatorenal syndrome (ICD-9: 572.4) & $1.139(1.055-1.230)$ & $<0.001$ \\
\hline Hepatic encephalopathy (ICD-9: 572.2) & $1.221(1.175-1.267)$ & $<0.001$ \\
\hline Ascites (ICD-9: 789.59) & $1.438(1.388-1.491)$ & $<0.001$ \\
\hline Biliary (ICD-9: 571.6) & $1.264(1.108-1.441)$ & $<0.001$ \\
\hline Bacterial infection & $1.018(0.981-1.055)$ & 0.35 \\
\hline Esophagogastroduodenoscopy & $0.793(0.760-0.828)$ & $<0.001$ \\
\hline Alcohol and drug rehabilitation & $0.862(0.775-0.958)$ & 0.006 \\
\hline
\end{tabular}

readmission were related to liver disease $(25.7 \%)$ and substance abuse (16.9\%). Hepatitis (7\%), alcohol-related diseases $(5.8 \%)$, and sepsis (5.4\%) were also in the top five causes of readmission in these patients.

\section{Discussion}

In our investigation of the largest US all-payer database for readmissions, we found a $31.4 \%$ readmission rate for patients with cirrhosis between 2010 and 2014, which is substantially higher than the national average readmission rate of $11 \%$ to $14 \%$ for any other medical condition $(16,17)$. It is also higher than the $18.8 \%$ rate reported for patients with cirrhosis from a similar database in the state of California (10) but is similar to the rate reported in another single-center study (27.0\%) (18). The readmission rate 
Table 5 Most common causes of readmission for patients with cirrhosis

\begin{tabular}{lc}
\hline Primary diagnosis of readmission & $\begin{array}{c}\text { Percentage of } \\
\text { patients }\end{array}$ \\
\hline Liver diseases-not otherwise specified & 25.7 \\
Substance abuse & 16.9 \\
Hepatitis & 7 \\
Alcohol-related disease & 5.8 \\
Sepsis & 5.4 \\
Gastrointestinal tract hemorrhage & 3.9 \\
Renal failure & 2.6 \\
Peritonitis & 2.1 \\
Fluid and electrolyte disorders & 2.1 \\
Graft complication & 1.6 \\
Pleurisy, pneumothorax, or pulmonary collapse & 1.3 \\
Skin or subcutaneous infections & 1.2 \\
Congestive heart failure & 1.2 \\
Surgical complications & 1.1 \\
Cancer or intrahepatic bile ducts & 1 \\
\hline
\end{tabular}

in our study, however, is much lower than that reported in another study, which indicated percentages as high as $50 \%$ among patients with decompensated cirrhosis (19). Moreover, we noted no annual increase in the number of readmissions during our study period. The most common reason for readmission in our study ( $\approx 25 \%)$ was liver diseases-not otherwise specified.

The burden of chronic medical conditions complicates the management of all patients and the adverse impact of any comorbidity on the care of cirrhotic appears to be profound. In our study, we noticed that many patients with readmissions had a higher number of concomitant comorbidities. Approximately $>30 \%$ of patients who had comorbidities like CHF, Peripheral vascular disease, COPD, peptic ulcer disease, diabetes mellitus with and without complications, and acquired immunodeficiency syndrome were readmitted. Our results highlight the impact of comorbid conditions on cirrhosis and raise the importance of multimorbidities on an individual basis during the index admission to prevent readmission. The association of these comorbidities with potentially preventable hospitalizations is clinically relevant because some of these conditions can respond to effective outpatient management. Hence, further research is needed to identify and characterize comorbidities and combinations of comorbidities that place patients at the highest risk for adverse outcomes.

With regard to patient-related predictors of readmission in our study, insurance status significantly affected the risk of readmission. The analysis of the payer status and trends in readmission of our study showed that being on Medicaid was associated with higher odds of readmission compared to Medicare patients. In contrast, the uninsured patients, those who paid out of pocket or those who had private insurance were associated with lower readmission rates compared with patients with Medicare insurance. The odds for readmission in the uninsured patients were slightly lower compared to patients with private insurance or Medicare group. However, our analysis revealed no relationship with socioeconomic factors like household income, in contrast with another study (11) that reported education level as predictive of readmission.

As higher 30-day readmission rates constitute a considerable burden on the nation's healthcare budget, a nationwide program like the Hospital Readmissions Reduction Program (HRRP) was implemented to tackle this issue. HRRP led to substantial reductions in readmission within 30 days of discharge among fee-for-service Medicare beneficiaries aged $>65$ years who were hospitalized with acute myocardial infarction, $\mathrm{CHF}$, or pneumonia-the target population for this program (20). The exceptionally high 30-day readmission rates related to CLD suggest that a similar program is very much needed to be modeled for these patients too.

It has been observed that post-discharge care and interventions in patients with chronic diseases significantly reduce the readmission rate, for example, one study looking at the trends of readmission in patients with COPD, postdischarge support considerably reduced the readmission rate within 30 days after discharge from hospital (21). Despite increased readmission rates for CLD than for CHF or COPD at 30 days, a significantly lower number of CLD hospitalizations had post-discharge care (skilled nursing facility, rehabilitation, or home health) $(13.2 \%$ vs. $27.4 \% \mathrm{CHF}$ and $23.2 \% \mathrm{COPD}, \mathrm{P}<0.01$ ), prompting us to acknowledge the need for post-discharge care for patients with CLD (5). As shown in our study, disposition status after index hospitalization significantly affected the readmission rate. Discharge to smaller hospitals or rehabilitation centers were associated with lower readmission rate compared with discharge home or leaving against medical advice. It implies that continuing to provide medical support in 
a healthcare setting decreased the rate of readmission. It is in contrast with the observations of Seraj et al., who observed no influence of disposition status on the rate of readmission (11). Nevertheless, they acknowledged the need for continued patient support based on their observation that the risk of readmission remains high (30\%) even three months after discharge (11). This highlights the need to develop and implement strategies to ensure continued, longitudinal support to patients even after index discharge, as well as to facilitate close monitoring of patients to identify complications early, possibly in outpatient clinics, and to allow for appropriate interventions that will preclude the need for readmission.

We observed in our study that readmitted patients also had higher incidence of associated secondary diagnoses of acute kidney injury and infection which is consistent with a recent study from Dallas-Fort Worth. That study looked at the time trends in CLD-related hospitalizations and concluded that although admissions for traditional complications of portal hypertension (ascites, varices, and peritonitis) remained stable over time from 2000-2015, however, hospitalization with complications related to infection increased by $11.7 \%$, and renal failure increased by sevenfold (22). This underscores the importance of redefining traditional cirrhosis associated complications, which will help refocus our future management.

In the current study, alcoholic cirrhosis carried a lesser risk of readmission than biliary or nonalcoholic cirrhosis, which is in contrast to another study that reported an increased risk of readmission among patients with alcoholic cirrhosis (10). The reason for this discrepancy is not apparent, but attending an alcohol rehabilitation program after discharge, with closer monitoring, may have a role-patients in our study who attended rehabilitation programs had a lower risk of readmission than those who did not. HE and ascites on index admission also were closely associated with the risk of readmission in our study, which is consistent with several previous studies $(6,11,23,24)$. Among the various age groups, patients older than 84 years had the lowest risk of readmission. Possible explanations for this may be that these patients are more likely to be discharged to a rehabilitation facility with closer monitoring or that more of these patients might have succumbed to death within the first 30 days after discharge or discharged to hospice. Further studies are necessary to investigate this observation.

Identifying factors that are modifiable during the index admission is essential for developing effective management strategies to minimize the risk of readmission in CLD. Interestingly, in our study, patients who underwent EGD during index hospitalization had lower rates of readmission than those who did not. It is possible that the use of EGD allowed physicians to identify high-risk varices during the index admission and subsequent intervention, which thereby decreased the chances of re-bleeding and readmission. A similar beneficial effect of timely paracentesis has been reported to reduce the risk of readmission in patients with cirrhosis-related ascites (25). These findings highlight the importance of thorough workup in patients with cirrhosis during any given hospitalization to identify possible risk factors for future complications and take appropriate remedial measures. Non-alcoholic fatty liver disease (NAFLD) can progress to cirrhosis is and a significant cause of cryptogenic cirrhosis. Literature has shown an increase in the prevalence of NAFLD (26). After reviewing data over six years (2011-2017) from multiple datasets, the American Society for Metabolic and Bariatric Surgery (ASMBS) found that the rate of bariatric surgery in patients with NAFLD who fail initial lifestyle interventions is also increasing (https://asmbs.org/resources/estimate-of-bariatric-surgerynumbers\#). The published literature on bariatric surgery has shown increased hospital LOS and mortality rates in compensated cirrhosis compared to patients without cirrhosis (these parameters are even worse in patients with decompensated cirrhosis who have bariatric surgery) (27), there is very little literature on readmissions in cirrhotic patients with previous bariatric surgery. We, therefore, decided to look at this subset of patients and found that previous bariatric surgery was associated with increased odds of readmission. The cause for this observation is unclear and bears further study, especially as there is a dearth of studies assessing the long-term outcomes of patients with cirrhosis and post-bariatric surgery.

Although the need for prevention of readmissions is widely recognized, the current management strategies for reducing cirrhosis-related readmissions appear to be far from satisfactory. Some investigators believe that as many as one-third of readmissions in cases of cirrhosis may be preventable $(18,28)$. Therefore, implementing appropriate measures may decrease the economic and healthcare burden caused by readmission in patients with cirrhosis. Several strategies have been proposed for preventing readmission among cirrhosis patients. Tapper et al. showed that measures to improve the quality of treatment by using an electronic decision support checklist for $\mathrm{HE}$, close monitoring, and prophylactic treatment for spontaneous bacterial peritonitis 
helped decrease readmission rates (29). Patient education is another critical area of intervention; Garrido et al. showed that providing even a 15 -minute education session regarding HE could significantly decrease the risk of readmission (30). We believe that our study, with data retrieved from a nationwide, all-payer database, can be useful for developing appropriate proactive strategies to decrease readmission rates.

Although this was a comprehensive study encompassing data from the entire country, it has some limitations. Our study could be affected by missing data and data misrepresentation, given the retrospective study design and the dependence on the database that use patients' codified electronic health records. Although the diagnostic codes for cirrhosis and its complications, except for that of ascites, have a relatively high positive predictive value and overall accuracy, using ICD-9 codes cirrhosis alone may have decreased sensitivity and need to be combined with ICD-9 codes for cirrhosis complications. In addition, the ICD-9 code used for biliary cirrhosis (571.6) is often associated with early-stage PBC or PSC instead of cirrhosis (31). This study did not account for some known significant predictors of readmission, such as patient-specific information including laboratory values, prognosis scores like the Maddrey Discriminant Function or Model for End-Stage Liver Disease, procedural details, or treatment administered during the index admission.

\section{Conclusions}

This study used the NRD to determine the incidence and predictors of cirrhosis-related 30-day readmissions between 2010 and 2014. We found that although there is no change in the readmission rate during the study period, rates of 30 -day readmissions are as high as $31.4 \%$. We identified several modifiable and non-modifiable factors that appear to increase the risk of 30-day readmission among patients hospitalized for cirrhosis, which have various implications, especially for the Medicare, enforced hospital readmission related penalties. We would also like to raise the importance of adding CLD/cirrhosis in the diagnostic/prognostic models for chronic medical conditions. The results of this study also stress the fact that certain non-traditional diagnoses such as infections, renal failure, and HE are becoming responsible for the high rate of readmissions. We believe that the insights drawn from this large-scale, nationwide study could inform healthcare policymakers and aid the development of guidelines for effective management of cirrhosis to reduce the burden of early readmission.

\section{Acknowledgments}

Funding: None.

\section{Footnote}

Reporting Checklist: The authors have completed the STROBE reporting checklist. Available at https://dx.doi. org/10.21037/atm-20-1762

Data Sharing Statement: Available at https://dx.doi. org/10.21037/atm-20-1762

Conflicts of Interest: All authors have completed the ICMJE uniform disclosure form (available at https://dx.doi. org/10.21037/atm-20-1762). The authors have no conflicts of interest to declare.

Ethical Statement: The authors are accountable for all aspects of the work in ensuring that questions related to the accuracy or integrity of any part of the work are appropriately investigated and resolved. The study was conducted in accordance with the Declaration of Helsinki (as revised in 2013).

Open Access Statement: This is an Open Access article distributed in accordance with the Creative Commons Attribution-NonCommercial-NoDerivs 4.0 International License (CC BY-NC-ND 4.0), which permits the noncommercial replication and distribution of the article with the strict proviso that no changes or edits are made and the original work is properly cited (including links to both the formal publication through the relevant DOI and the license). See: https://creativecommons.org/ licenses/by-nc-nd/4.0/.

\section{References}

1. Asrani SK, Devarbhavi H, Eaton J, et al. Burden of liver diseases in the world. J Hepatol 2019;70:151-71.

2. Scaglione S, Kliethermes S, Cao G, et al. The Epidemiology of Cirrhosis in the United States. J Clin Gastroenterol 2015;49:690-6.

3. Allen AM, Kim WR, Moriarty JP, et al. Time trends in the health care burden and mortality of acute on chronic liver failure in the United States. Hepatology 2016;64:2165-72.

4. Desai AP, Reau N. The Burden of Rehospitalization 
for Patients With Liver Cirrhosis. Hosp Pract (1995) 2016;44:60-9.

5. Asrani SK, Kouznetsova M, Ogola G, et al. Increasing Health Care Burden of Chronic Liver Disease Compared With Other Chronic Diseases, 2004-2013. Gastroenterology 2018;155:719-29.e4.

6. Di Pascoli M, Ceranto E, De Nardi P, et al. Hospitalizations Due to Cirrhosis: Clinical Aspects in a Large Cohort of Italian Patients and Cost Analysis Report. Dig Dis 2017;35:433-8.

7. Rajasingh CM, Tennakoon L, Staudenmayer KL. Selfinflicted gunshot wounds: readmission patterns. J Surg Res 2018;223:22-8.

8. Weinreich MA, Styrvoky K, Chang S, et al. Sepsis at a Safety Net Hospital: Risk Factors Associated With 30-Day Readmission. J Intensive Care Med 2019;34:1017-22.

9. Patel B, Sablani N, Garg J, et al. Thirty-day readmissions after cardiac implantable electronic devices in the United States: Insights from the Nationwide Readmissions Database. Heart Rhythm 2018;15:708-15.

10. Wei M, Ford J, Li Q, et al. Hospital Cirrhosis Volume and Readmission in Patients with Cirrhosis in California. Dig Dis Sci 2018;63:2267-74.

11. Seraj SM, Campbell EJ, Argyropoulos SK, et al. Hospital readmissions in decompensated cirrhotics: Factors pointing toward a prevention strategy. World J Gastroenterol 2017;23:6868-76.

12. Fagan KJ, Zhao EY, Horsfall LU, et al. Burden of decompensated cirrhosis and ascites on hospital services in a tertiary care facility: time for change? Intern Med J 2014;44:865-72.

13. Morales BP, Planas R, Bartoli R, et al. Early hospital readmission in decompensated cirrhosis: Incidence, impact on mortality, and predictive factors. Dig Liver Dis 2017;49:903-9.

14. Chirapongsathorn S, Krittanawong C, Enders FT, et al. Incidence and cost analysis of hospital admission and 30-day readmission among patients with cirrhosis. Hepatol Commun 2018;2:188-98.

15. Ganesh S, Rogal SS, Yadav D, et al. Risk Factors for Frequent Readmissions and Barriers to Transplantation in Patients with Cirrhosis. PLoS One 2013;8:e55140.

16. Berry JG, Gay JC, Joynt Maddox K, et al. Age trends in 30 day hospital readmissions: US national retrospective analysis. BMJ 2018;360:k497.

17. Fingar KR BM, Jiang JH. A Comparison of All-Cause
7-Day and 30-Day Readmissions, 2014. In: Healthcare Cost and Utilization Project (HCUP) Statistical Briefs. Agency for Healthcare Research and Quality. Available online: www.hcup-us.ahrq.gov/reports/statbriefs/ sb230-7-Day-Versus-30-Day-Readmissions.pdf. Accessed March 2018.

18. Agrawal K, Kumar P, Markert R, et al. Risk Factors for 30-Day Readmissions of Individuals with Decompensated Cirrhosis. South Med J 2015;108:682-7.

19. Scaglione SJ, Metcalfe L, Kliethermes S, et al. Early Hospital Readmissions and Mortality in Patients With Decompensated Cirrhosis Enrolled in a Large National Health Insurance Administrative Database. J Clin Gastroenterol 2017;51:839-44.

20. Zuckerman RB, Sheingold SH, Orav EJ, et al. Readmissions, Observation, and the Hospital Readmissions Reduction Program. N Engl J Med 2016;374:1543-51.

21. Pedersen PU, Ersgard KB, Soerensen TB, et al. Effectiveness of structured planned post discharge support to patients with chronic obstructive pulmonary disease for reducing readmission rates: a systematic review. JBI Database System Rev Implement Rep 2017;15:2060-86.

22. Asrani SK, Hall L, Hagan M, et al. Trends in Chronic Liver Disease-Related Hospitalizations: A PopulationBased Study. Am J Gastroenterol 2019;114:98-106.

23. Atla PR, Sheikh MY, Gill F, et al. Predictors of hospital re-admissions among Hispanics with hepatitis C-related cirrhosis. Ann Gastroenterol 2016;29:515-20.

24. Tapper EB, Halbert B, Mellinger J. Rates of and Reasons for Hospital Readmissions in Patients With Cirrhosis: A Multistate Population-based Cohort Study. Clin Gastroenterol Hepatol 2016;14:1181-8.e2.

25. Le S, Spelman T, Chong CP, et al. Could Adherence to Quality of Care Indicators for Hospitalized Patients With Cirrhosis-Related Ascites Improve Clinical Outcomes? Am J Gastroenterol 2016;111:87-92.

26. Younossi ZM, Stepanova M, Afendy M, et al. Changes in the prevalence of the most common causes of chronic liver diseases in the United States from 1988 to 2008. Clin Gastroenterol Hepatol 2011;9:524-30.e1; quiz e60.

27. Mosko JD, Nguyen GC. Increased perioperative mortality following bariatric surgery among patients with cirrhosis. Clin Gastroenterol Hepatol 2011;9:897-901. 
28. Volk ML. How can we improve quality of care for patients with cirrhosis? Gastroenterology 2012;143:17-9.

29. Tapper EB, Finkelstein D, Mittleman MA, et al. A Quality Improvement Initiative Reduces 30-Day Rate of Readmission for Patients With Cirrhosis. Clin Gastroenterol Hepatol 2016;14:753-9.

30. Garrido M, Turco M, Formentin C, et al. An

Cite this article as: Garg SK, Goyal H, Obaitan I, Shah PA, Sarvepalli S, Jophlin LL, Singh D, Asrani S, Kamath PS, Leise MD. Incidence and predictors of 30-day hospital readmissions for liver cirrhosis: insights from the United States National Readmissions Database. Ann Transl Med 2021;9(13):1052. doi: 10.21037/atm-20-1762 educational tool for the prophylaxis of hepatic encephalopathy. BMJ Open Gastroenterol 2017;4:e000161.

31. Nehra MS, Ma Y, Clark C, et al. Use of administrative claims data for identifying patients with cirrhosis. J Clin Gastroenterol 2013;47:e50-4. 
Appendix 1

Title: ICD-9 codes for Charlson Comorbidity Index Conditions

\begin{tabular}{|c|c|}
\hline Diagnosis & ICD-9-CM \\
\hline Congestive heart failure & $428,398.91,402.01,402.11,402.91,404.01,404.03,404.11,404.13,404.91,404.93,425.4-425.9$ \\
\hline Peripheral vascular disease & $443.9,441 . x, 785.4$, V43.4, Procedure 38.48 \\
\hline Cerebral vascular accident & $430 . x-438 . x$ \\
\hline Pulmonary disease & $490 . x-505 . x, 506.4$ \\
\hline Connective tissue disorder & $710.0,710.1,710.4,714.0,714.1,714.2,714.8,725 . x$ \\
\hline Peptic ulcer & 531.x-534.x \\
\hline Liver disease & $571.2,571.4,571.5,571.6$ \\
\hline Paraplegia & 342.x, 344.1 \\
\hline Renal disease & 582.x, 583.0, 583.1, 583.2, 583.3, 583.5, 583.6, 583.7, 583.4, 585, 586, 588 \\
\hline Cancer & $\begin{array}{l}14,15,16,18,170,171,172,174,175,176,179,190,191,192,193,194,1950,1951,1952,1953, \\
1954,1955,1958,200,201,202,203,204,205,206,207,208\end{array}$ \\
\hline Metastatic cancer & $196,197,198,1990,1991$ \\
\hline Severe liver disease & $572.2,572.3,572.4,572.8$ \\
\hline Human immunodeficiency virus & $042,043,044$ \\
\hline
\end{tabular}

